\title{
Suitable Image Features for Drought Stress Detection in Beans using Raspberry PI Imaging System
}

\author{
Daniel Maitethia Memeu \\ Department of Physical Sciences, Faculty of Physics \\ Meru University of Science and Technology, Kenya \\ Amos Chege Kirongo \\ Department of Computer Science and IT \\ Meru University of Science and Technology, Kenya \\ Richard Boiyo \\ Department of Physical Sciences, Faculty of Physics \\ Meru University of Science and Technology, Kenya \\ Alex M Kimuya \\ Department of Physical Sciences, Faculty of Physics \\ Meru University of Science and Technology, Kenya
}

\begin{abstract}
Crop stresses are often detected late and this leads to massive crop yield loss. There exists a need for development of a real time system for monitoring crop growth progress with a view of early detection of diseases and other forms of crop stress. In this work, a simple raspberry pi imaging system is developed and used to capture images of beans subjected to drought stress. The images are used to extract features for classification of the stress. The features are then used to train classifiers for drought stress detection. Detection accuracy of between 90 to $100 \%$ has been achieved.
\end{abstract}

Key Words:Draught stress; Raspberry Pi; Segmentation; Computer vision; Artificial Neural Network; MATLAB

\section{INTRODUCTION}

Crop stress is any factor that causes unfavorable growth conditions in crops. Water drought is one form that adversely affects crop productivity [1]. It occurs when the loss of water by transpiration exceeds that absorbed from the soil. Severe drought stress has direct negative impacts on the quantity and quality of crop yield. It limits crop productivity and yield by reducing photosynthesis and leaf growth. If it remains undetected for a long time, the entire crop could be lost.

This loss can be prevented by early detection and watering the crop at appropriate times. The common method of detection of drought stress in crops has been visual assessment of symptoms such as withering. However, such assessment is qualitative and subjective and may occur too late when physiological damage of crops has taken place. To address this problem, a quantitative method of crop drought stress detection ought to be developed.

In the past, different methodologies have been proposed by different researchers for early detection of drought stress in crops [2], [3]. The common approach has been the use of thermography and hyperspectral vision [4]. Thermography utilizes infrared thermometer sensors or thermal cameras to measure the canopy temperature $\left(T_{c}\right)$ and to define crop water stress indexes [5], [6]. However, $T_{c}$ measurement presents low resolution and it is susceptible to meteorological conditions and foliage geometric structure such as leaf angles [7]. Furthermore the process is intrusive to crops and the measurement procedure is time consuming. On the other hand, hyperspectral analysis consists of monitoring changes in the chlorophyll fluorescence or in photochemical reflectance. The process requires successive monitoring of crops using sophisticated multispectral imaging systems [7]. 
Computer vision is one attractive alternative for monitoring crop progress in the field. This is due to availability of cheap but high quality cameras and powerful computing hardware such as Raspberry Pi. Besides, advanced machine learning and computer vision algorithms have been developed and implemented in various computer programming languages and are available as open source libraries.

A number of studies attempting to use image processing techniques to detect crop stress have been reported [2], [8]. However, the proposed solutions often oversimplify the problem making them impractical. For instance, a number of studies assume a controlled greenhouse condition [9]. Use of different hardware setups and fundamentally different algorithms for monitoring different crop stresses is also another problem in the reported work [10], [11]. For the solution to be optimal, it should be easily adaptable in different situations with minimal changes. For example a computer vision system for monitoring water stress in beans should also be capable of monitoring and detecting nutritional or pathogen stress in beans or other crops without major modification.

For this ideal case to be achieved, two things ought to be done. One, image features to be used as training set in classifiers used for detecting different crop stresses ought to be investigated. Second, Classifiers for detecting crop stress ought to be trained using suitable image features. After all the classifiers have been trained and the imaging system has been set up to acquire the images, appropriate image features would then be extracted and relevant classifier invoked to perform the detection of possible crop stress.

The aim of this study was to investigate suitable image features to be used in early detection of beans drought stress under uncontrolled environmental conditions. Digital images of beans top canopy were obtained from crops subjected to drought stress and healthy crops at periodic intervals, preprocessed, and various features extracted. An Artificial Neural Network classifier was then trained and used to detected crop drought stress using the extracted image features. The detection accuracy of the classifier was then accessed.

\section{METHODOLOGY}

An imaging device was assembled and programmed to capture RGB images at a periodic intervals of 15 minutes upon power up. The device was used to capture images of beans crop which were planted and a portion of them subjected to draught stress. The images were captured continuously from the point when the crops were subjected to the stress up to the point when the stress was visually manifested through withering. Different image features of the crops under investigation were extracted and analyzed to determine the most suitable features to aid in early detection of drought stress.

\subsection{Assembly and programming of the imaging system}

\subsubsection{Hardware}

The hardware components of the digital image processing system comprised of a 2592 x 1944 pixels Raspberry pi camera module (imaging sensor), raspberry pi (a 700MHz system on chip microcomputer), 8GB micro-SD card for storing the acquired images, a USB powered hub, and solar power supply. The Raspberry pi camera module was mounted to the Raspberry pi and the power of the system was supplied by a solar power supply (comprising of $100 \mathrm{~W}$ panel, 100AH lead acid battery and a 2.5A charge controller). A powered USB hub was used to convert the $12 \mathrm{~V}$ from the output of the power supply to $5 \mathrm{~V}$ suitable for powering the Raspberry pi. The block diagram of the setup is depicted in Figure 1.

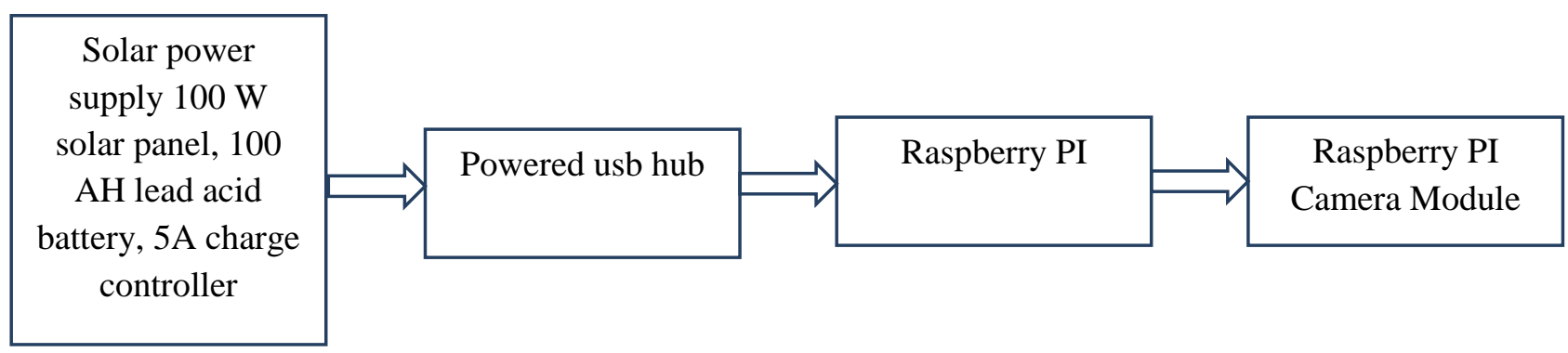

Figure 1: Block Diagram showing the Imaging system 


\subsubsection{Software}

A Linux script for performing time lapse photography was written to run in the raspberry pi. The interval of capturing images was set to 15 minutes. The task scheduler of the Raspberry pi was configured to always run the script every time the pi was powered on. Images captured were named according to the order in which they were acquired. The flow chart of the image acquisition logic is shown in Figure 2.

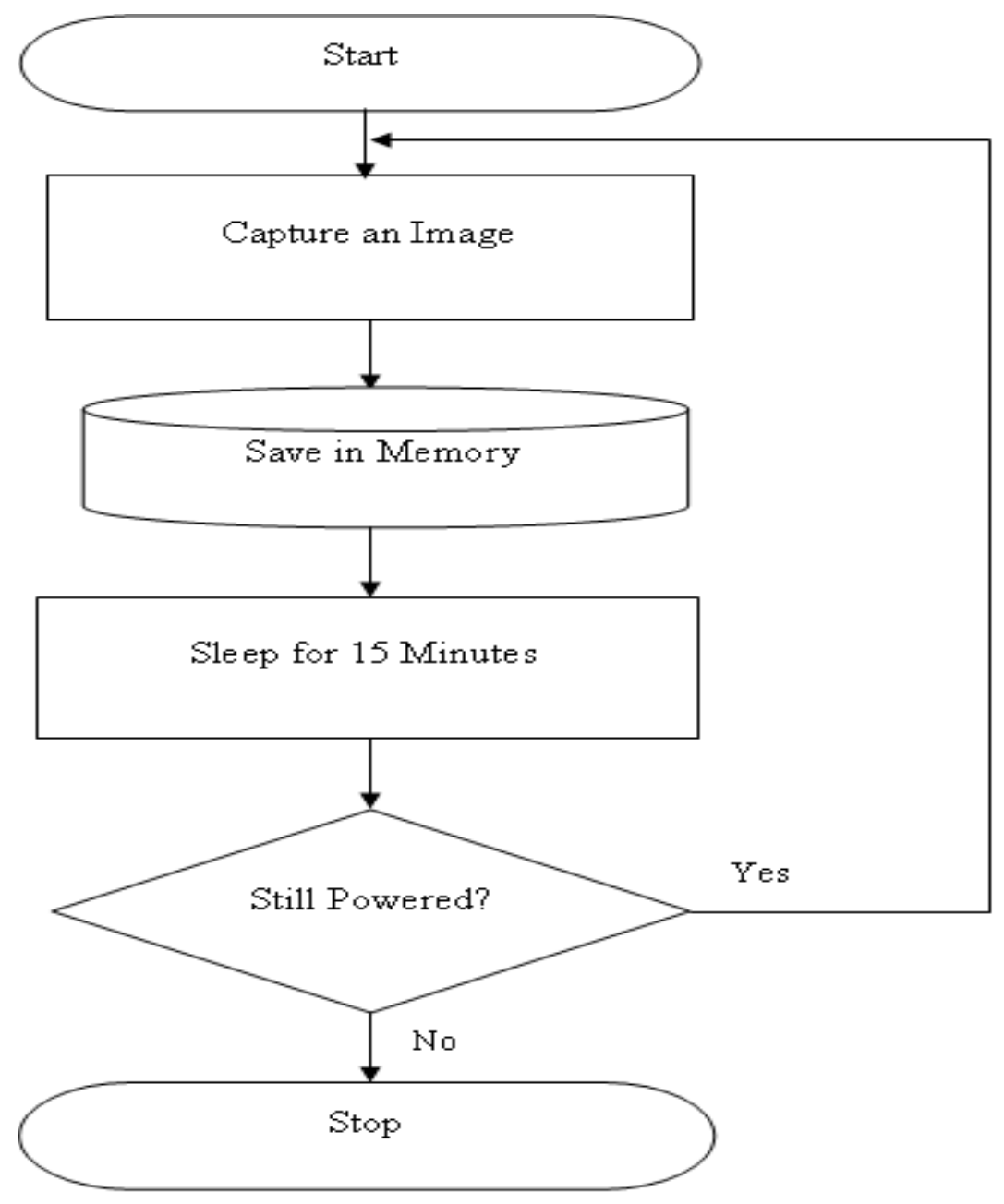

Figure 2: Logic diagram of the image acquisition device

\subsection{Growing of beans}

One type of bean variety (Rose coco) was used in this work to represent other bean varieties commonly grown. Two hundred $(10 \mathrm{~cm} \mathrm{X} 25 \mathrm{~cm})$ seed bags were filled with fertile soil and put in a greenhouse. In each bag, three seeds were planted and uniformly watered till germination. No attempt was made to control environmental conditions such as temperature, relative humidity and sun light intensity thus providing a natural environment for plant growth.

\subsection{Image acquisition}

Upon germination beans seeds, the seedlings were subdivided into two equal sets with each set comprising of one hundred seed bags with each bag having three seedlings. One set of the crops was adequately watered daily and this formed the health set (control experiment) while the other set was subjected to drought stress by completely being 
denied water and this formed the stressed set (test set). The assembled imaging system was mounted vertically above the crop canopy at a height of two and half meters such that the field of view covered both sets of crops. The imaging system was then powered to start the image acquisition process. This process continued daily, till the stress was visually seen on the fourth day. Figure 3 shows the setup of the bean crops with the two sets labeled.

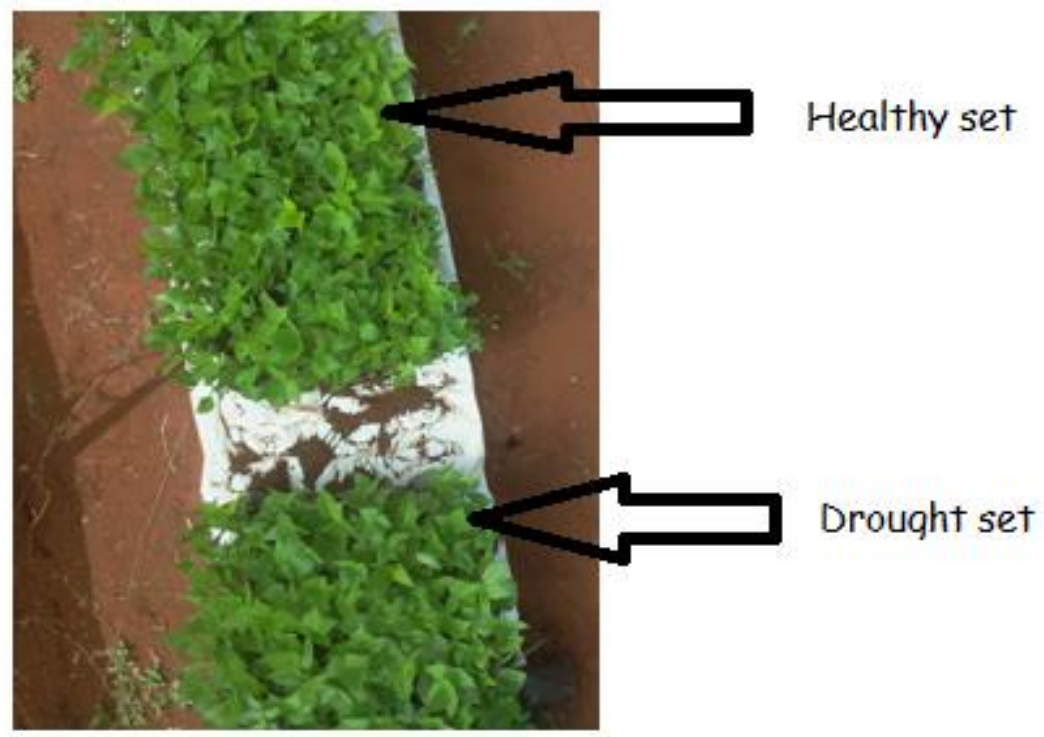

Figure 1: Beans crop setup

\subsection{Image processing, classification and analysis}

\subsubsection{Image Preprocessing}

Preprocessing consisted of two steps: First, manual removal of unclear night images and secondly image cropping. Three sub-images were cropped from each captured image as follows; a sub-image each from both healthy and drought sets and a reference image from a bare surface. Cropping of images was done by selecting a sample image and identifying image coordinates representing the three regions. Using these coordinates, 200 square pixel subimages were extracted from the original images. The cropped images were saved into three different directories in a computer.

A total of 330 crop images were captured for four days. Half of these images were captured at nights and contained no meaningful information due to darkness in the greenhouse and so they were discarded. Each of the remaining images was cropped into three sub-images to yield a total of 495 images corresponding to health, stressed and reference sets. Figure 4 shows sample images of the original and cropped sets. 


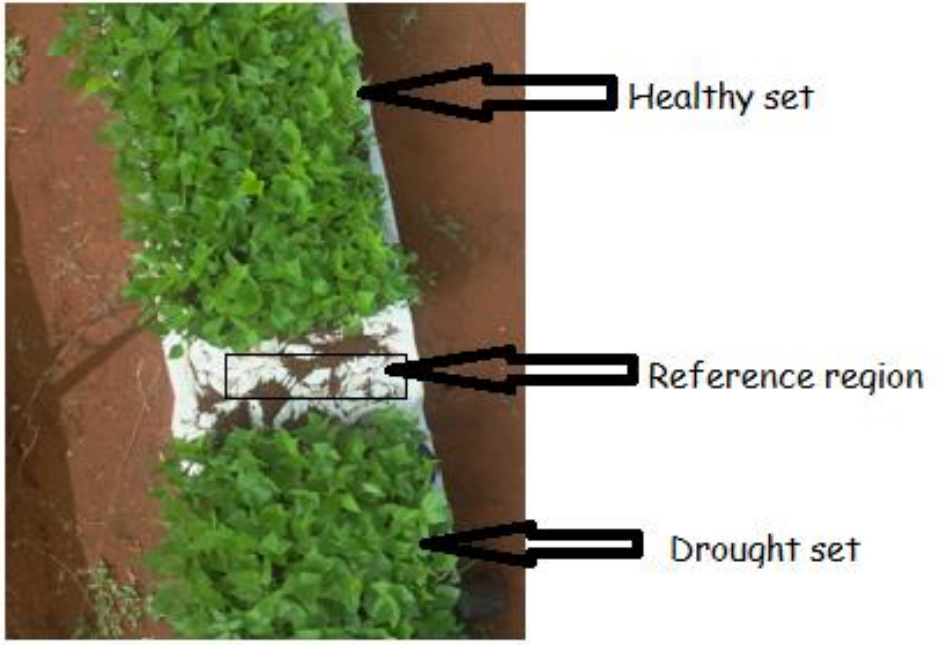

a
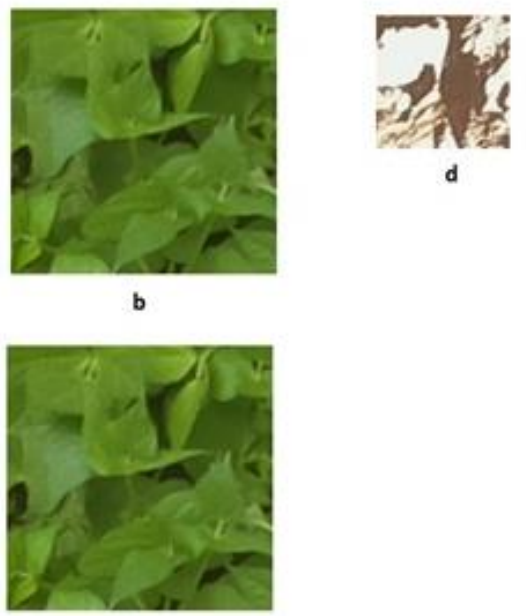

c

Figure 4: Sample images, a) raw image, b) crop image from the health set, c) cropped image from the stressed set, d) reference image

Visual evaluation revealed that the stressed set of crops manifested clear signs of withering in the fourth day of the experiment. Figure 5 shows both the health and stressed sets of images captured during the four days.

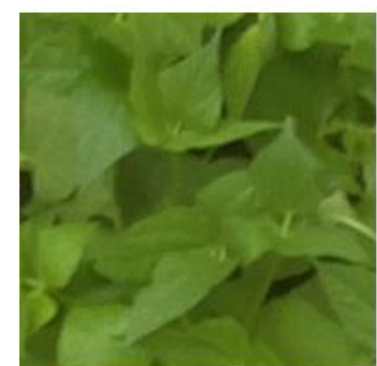

a

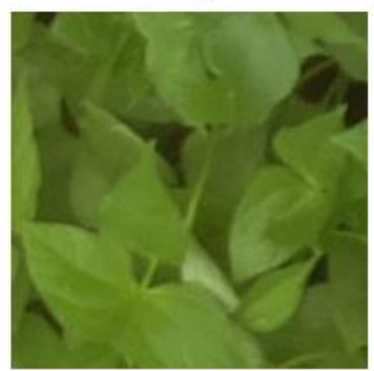

e

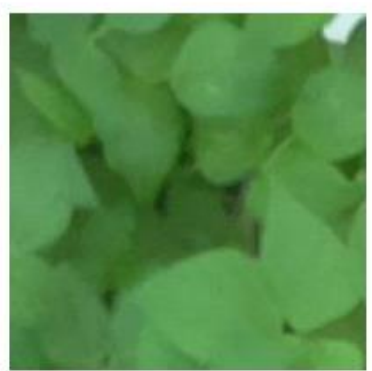

b

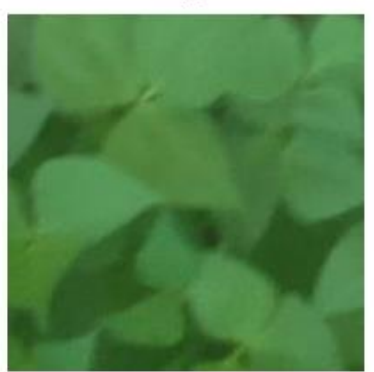

f

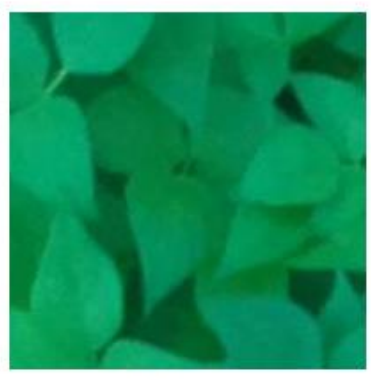

c

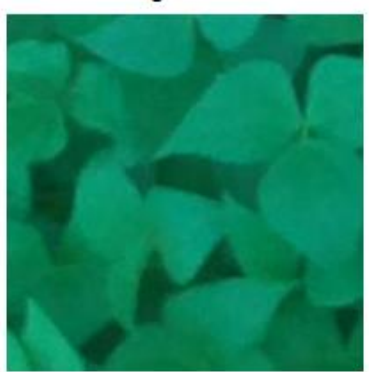

g

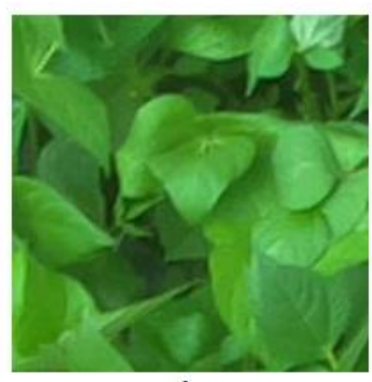

d

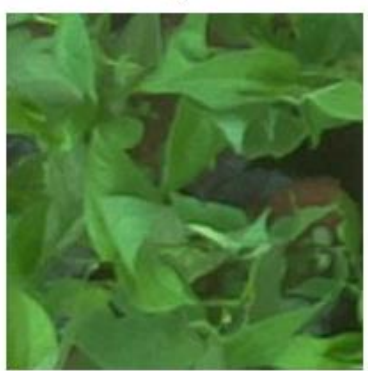

h

Figure 5: Health (a, b, c, d) and stressed (e, f, g, h) crop image samples captured for four days

From figure 5, it is difficult to visually identify signs of drought stress from images captured during the first three days. The difference in the image appearance was due to variations in light intensity during times in the day.

\subsection{Feature Extraction}




\subsubsection{Averaged Image Intensities of different $R G B$ components}

The mean intensities $\left(I_{m}\right)$ for the (R, G, and B color components) of each of the cropped sub images were computed. Variations in day light intensities were corrected by subtracting the RGB means of reference images from the corresponding RGB images of both the health and stressed images, i.e.

$$
I_{m e}=I_{m}-I_{m y}
$$

Where $I_{m e}$ is the corrected mean intensities for either the stressed or health plants images, $I_{m}$ is the mean of the raw image from either stressed or healthy plants and $I_{m y}$ is the mean intensities of the reference image. Time variations of the corrected mean intensities of individual color components (R,G, and B) for the stressed and health sub-images was performed to determine the component which gave the greatest variance.

\subsection{Classification using ANN}

The averaged image intensities from the two sets of images (healthy and drought sets) were used in training and validation of a multilayered Artificial Neural Network (ANN) classifier. The objective was to feed the ANN with the intensity features from either healthy or drought set of crop images and then get an output indicating whether the crop from which the input features were obtained is healthy or drought stressed. Eighty percent of the intensity features were used in training ANN while $20 \%$ of the features were used to access the classification accuracy of the trained classifier. Different numbers of hidden neuron of the ANN were used during the training session and the classifiers were saved. The classifier which recorded the best learning performance was used in the validation session using an independent set of features. MATLAB ANN Toolbox was used to create and train the ANN classifier. The training set comprised of 330 feature vectors from 165 images captured and a series of 1's and 0's as the targets (desired outputs) corresponding to feature vectors from health and stressed plant images respectively.

\subsection{Evaluation of the plant rate of growth}

The rate of growth for both stressed and health plant was also accessed. This was done by segmenting the green component of the RGB images captured from the two sets of plants to obtain the top canopy of the crops as the foreground of the images. The purpose of segmentation was to partition the top leaves of the plants (foreground) from the rest of the image (background) and determine their area. A threshold value for segmenting each image was determined by multiplying the value obtained from Otsu's algorithm [12], [15], [16] by a factor of 1.2 (empirically determined). The total area of the segmented region for each image was then computed by summing the foreground pixels for each image. Daily rate of change of the area of the foreground regions (rate of growth) was performed for both stressed and health crop images using 29 images captured in each day. The algorithmic steps for this procedure is given in Table 1

Table 1: Algorithmic stems for monitoring the rate of growth of both stressed and health crops

1. Load RGB images (from stressed and health crops) into Matlab ${ }^{T M}$

2. Convert the images to gray scale by extracting the green component images

3. Threshold the gray scale image using a threshold value determined by Otsu's method and scaled by a factor of 1.2

4. Use regionprops function in Matlab ${ }^{T M}$ to determine the area of the foreground regions

5. Scatter the results obtain against the order in which the images were captured

6. Use Matlab ${ }^{T M}$ curve fitting tool to perform a linear fit and determine the gradient

7. Repeat the above procedure for the two sets of crops images for entire period the images were captured.

Figure 6 shows sample images of the segmented canopies for both stressed and health crop images. The region of interest was the top leaves because of their rapid growth rate which is a general indicator of the plant growth. Images regions from the top leaves of the plant have higher intensity values compared to regions with lower leaves. 


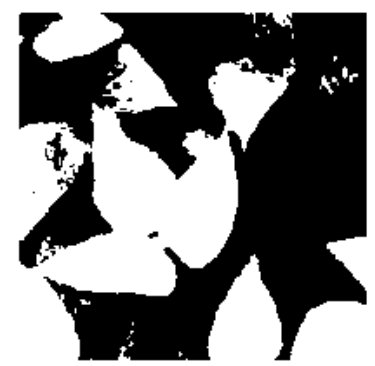

a

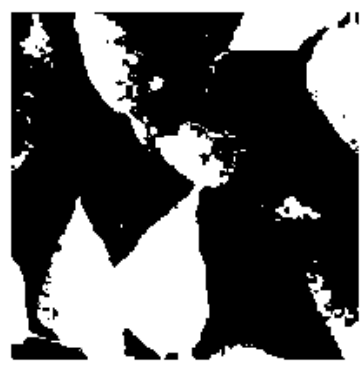

e

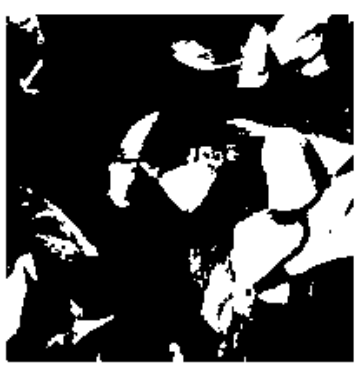

b

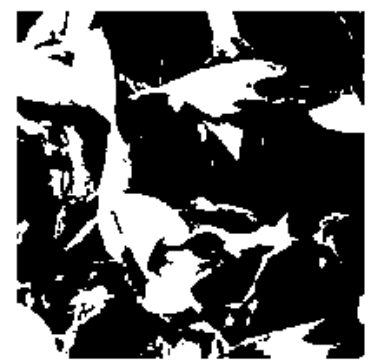

f

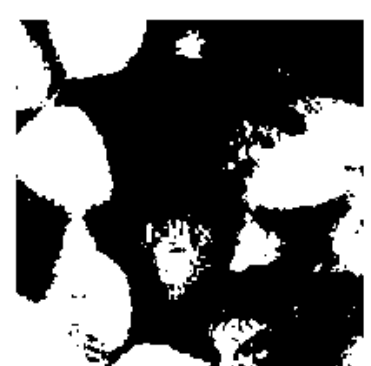

c

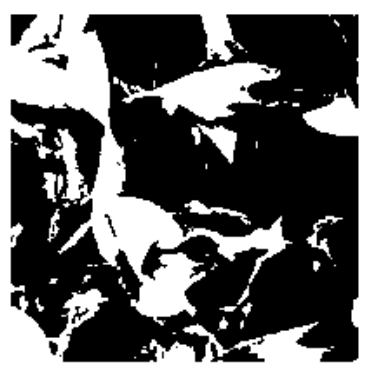

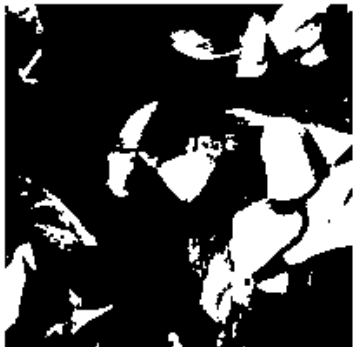

d

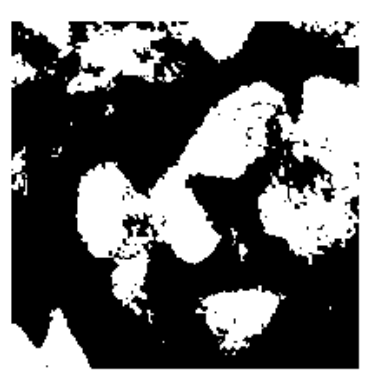

h

Figure 6: Segmented top canopy of health (a, b, c, and d) and stressed (e, f, g, and h) images

To assess the daily rate of growth for both stressed and health sets of plants, total area of segmented canopy for every image captured at different time intervals was computed and area rate of change evaluated. The area rate of change was computed by subtracting area of foreground regions obtained from images captured at successive intervals.

\section{RESULTS AND DISCUSSION}

\subsection{Averaged Image Intensity}

Figure 7 gives the time variation scatter plots for mean intensities for the Red, Green and Blue components. 

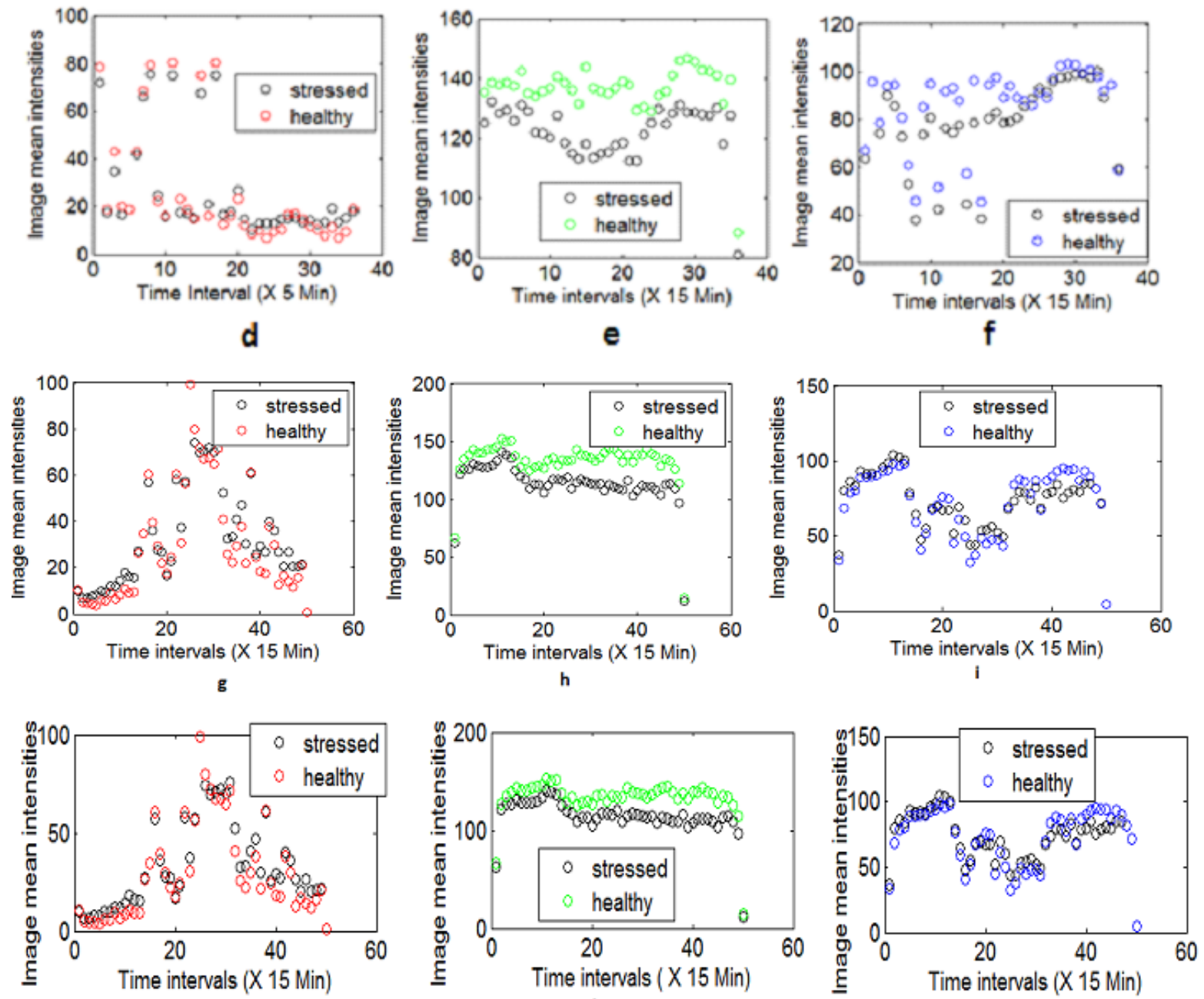

j
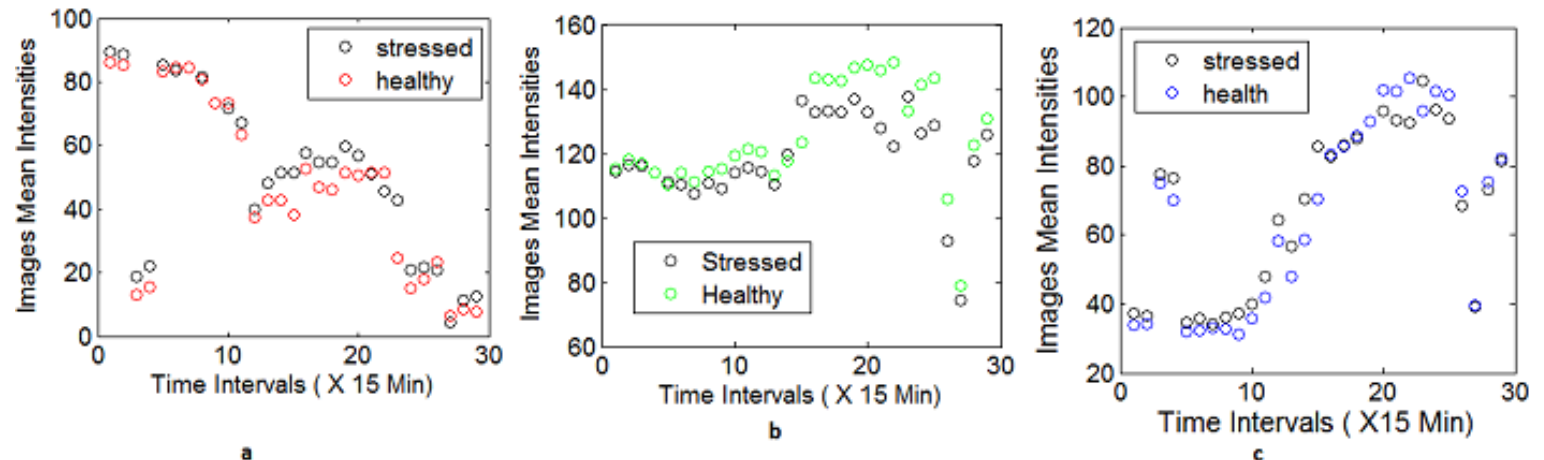

Figure 7: Time variations of mean intensities for Red (a, d, g and j), Green (b, e, h, and k) and Blue (c, f, i and l) color components

It can be seen from the scatter plots that the Green color component means indicate an elaborate decision boundaries at middle range of the plots between the stressed set and healthy set crops. The blue and red color components show no clear visual demarcations between the two crop sets. This implies that green color component mean could be a better discriminator of healthy and stressed crop images. 
International Journal of Innovations in Engineering and Technology (IJIET)

http://dx.doi.org/10.21172/ijiet.81.001

\begin{tabular}{lrrrrrrrrrrrrrrrr} 
Time & 1 & 2 & 3 & 4 & 5 & 6 & 7 & 8 & 9 & 10 & 11 & 12 & 13 & 14 \\
\hline day1 & 0.90 & 1.42 & 0.76 & 0.00 & -0.85 & 3.97 & 3.40 & 3.48 & 6.14 & 5.22 & 5.66 & 6.29 & 2.89 & -1.89 \\
day2 & 10.04 & 6.27 & 9.49 & 9.51 & 11.62 & 11.09 & 6.20 & 12.18 & 14.40 & 16.37 & 13.34 & 19.90 & 21.41 & 18.28 \\
day3 & 4.72 & 4.65 & 8.70 & 12.34 & 11.82 & 11.85 & 12.19 & 14.44 & 14.43 & 12.30 & 11.62 & 11.29 & 14.77 & 12.96 \\
day4 & 4.72 & 4.65 & 8.70 & 12.34 & 11.82 & 11.85 & 12.19 & 14.44 & 14.43 & 12.30 & 11.62 & 11.29 & 14.77 & 12.96 & \\
& & & & & & & & & & & & & & & & \\
\hline
\end{tabular}

Table 2: Differences in mean intensities for the health and stressed images

Table 2 shows the values of the difference between green color component mean intensity from health and stressed images. Positive values imply that the mean intensities for healthy set are greater than those for stressed set. In general it was observed than the mean color intensities for the health images was higher than those for the stressed. In day 1 there were five measurements out of 29 which deviated from this trend. However, from day 2 all the mean intensities of health images were greater than those of stressed images. This therefore implies that the magnitudes of the green component of the RGB image is a good indicator for drought stress monitoring.

\subsection{Image classification using ANN}

The performance of the ANN trained with different number of hidden neurons is given in Table 2. The table shows results for the training, and validation sessions as generated by MATLAB ANN toolbox. Training of the ANN used $40 \%$ of the data in the training set. Another $40 \%$ was used for validation while the remaining $20 \%$ was used of in the testing stage. Performance of ANN in the three sessions was measured in terms of correlation coefficient between the desired output and the system response.

\subsubsection{Performance of ANN Results}

\begin{tabular}{llll}
$\begin{array}{l}\text { Number } \\
\text { of hidden } \\
\text { neurons }\end{array}$ & $\begin{array}{l}\text { Training } \\
\text { (correlation }\end{array}$ & $\begin{array}{l}\text { Validation } \\
\text { (correlation } \\
\text { coefficient) }\end{array}$ & $\begin{array}{l}\text { Testing } \\
\text { (correlation } \\
\text { coefficient) }\end{array}$ \\
\hline 15 & 0.8642 & 0.7420 & 0.8326 \\
20 & 0.8872 & 0.7570 & 0.9000 \\
30 & 0.8646 & 0.7633 & 0.6743 \\
40 & 0.9090 & 0.7237 & 0.6349
\end{tabular}

Table 3: Performance of ANN

From table 3, its evident that best generalization was achieved by an ANN trained using 20 hidden neurons. This therefore implies that best classification accuracy of ANN trained to detect drought stress is $90 \%$.

\subsection{Rate of growth}

To assess the daily rate of growth for both stressed and health sets of plants, total area of segmented canopy for every image captured at different time intervals was computed and area rate of change evaluated. Table 4 gives the area rates of change of both the stressed and health images for four days. 


\begin{tabular}{lllcl} 
& & & Rate of growth & \\
& Day1 & Day2 & Day3 & Day4 \\
\hline Health set & 7 & 200 & 200 & 200 \\
Stressed set & -2.5 & -190 & -190 & -190
\end{tabular}

Table 4: Rate of growth for both health and stressed images

It is evident that positive rate of change was recorded for the health set for all the four days while the stressed set recorded a negative rate of growth. This implies that the total leave areas for the well watered crops continued to increase while those of the drought stressed set shrunk (negative growth). Hence, it can be concluded that the area rate of change of the plant top canopy can be a good indicator of drought stress.

\section{CONCLUSION AND FUTURE PERSPECTIVES}

In this work suitable image features for discriminating crops under drought stress from healthy crops using top canopy images captured under uncontrolled environmental conditions were investigated. Averaged image intensity from green components of RGB images captured. RGB color component mean intensities were extracted and analyzed. It was found that the green color component means for healthy set crops had magnitudes greater than those of stressed crops. This suggest that green color component image intensity of crops is a good feature for drought stress classification. ANN classifier trained with these features (green color component mean intensities) to distinguish between healthy and stressed crops yielded $90 \%$ classification accuracy. Finally, top canopy rate of growth for the crops was also investigated by computing the areas of top canopy leaves. It was found that while healthy crops recorded positive growth, drought subjected crops recorded negative growth. Future work should focus on investigating suitable crop image features to be used for early detection of other crop stress such as diseases and pest attacks.

\section{FUNDING}

This work was funded by Kenya Education Network (KENET) Raspberry Pi mini-grant and Meru University of Science and Technology

\section{ACKNOWLEDGEMENT}

We would wish to acknowledge Samuel Ntongai Lalai (Physics Technologist) for his invaluable technical support.

\section{REFERENCES}

[1] Behmann, J., Mahlein, A.-K., Rumpf, T., Römer, C., \& Plümer, L. (2015). A review of advanced machine learning methods for the detection of biotic stress in precision crop protection. Precision Agriculture, 16(3), 239-260. https://doi.org/10.1007/s11119-014-9372-7

[2] Marek Wójtowicz, Andrzej Wójtowicz, Jan Piekarczyk, Application of remote sensing methods in agriculture, COMMUNICATIONS IN BIOMETRY AND CROP SCIENCE VOL. 11, NO. 1, 2016, PP. 31-50

[3] Kirongo, C. A. (2016.). A Review of Image Processing Software Techniques for Early Detection of Plant Drought Stress. Retrieved from http://www.ijcat.com/archives/volume5/issue6/ijcatr05061009.pdf

[4] Fernandez-Jaramillo, A.A.; Duarte-Galvan, C.; Contreras-Medina, L.M.; Torres-Pacheco, I.;Romero-Troncoso, R.D.J.; Guevara-Gonzalez, R.G.; Millan-Almaraz, J.R. Instrumentation In Developing Chlorophyll Fluorescence Biosensing: A Review. Sensors 2012, 12, 1185311869.

[5] Agam, N.; Cohen, Y.; Berni, J.A.J.; Alchanatis, V.; Kool, D.; Dag, A.; Yermiyahu, U.; Ben-Gal, A.An Insight To The Performance Of Crop Water Stress Index For Olive Trees. Agric. Water Manag. 2013, 118, 79-86.

[6] Udompetaikul, V.; Upadhyaya, S.K.; Slaughter, D.; Lampinen, B.; Shackel, K.; House, G. Cropwater Stress Detection Using Leaf Temperature And Microclimatic Information. In Proceedingsof 2011 ASABE Annual International Meeting Sponsored By ASABE Galt House, Louisville,KY, USA, 7-10 August 2011.

[7] Ghulam, A., Li, Z.-L., Qin, Q., Yimit, H., \& Wang, J. (2008). Estimating crop water stress with ETM plus NIR and SWIR data. Agricultural and Forest Meteorology, 148(11), 1679-1695. https://doi.org/10.1016/j.agrformet.2008.05.020 
[8] David L. EHRET, Anthony LAU, Shabtai BITTMAN, Wei LIN, Tim SHELFORD, Automated monitoring of greenhouse crops, Agronomie 21 (2001) 403-414.

[9] Yufeng Ge, Geng Bai, Vincent Stoerger, James C. Schnable, Temporal dynamics of maize plant growth, water use, and leaf water content using automated high throughput RGB and hyperspectral imaging, Computers and Electronics in Agriculture ,127 (2016) 625-632.

[10] P. Symonds, A. Paap, K. Alameh, J. Rowe, C. Miller, A real-time plant discrimination system utilising discrete reflectance spectroscopy, Computers and Electronics in Agriculture, 117 (2015) 57-69.

[11] Geng Bai, Yufeng Ge, Waseem Hussain, P. Stephen Baenziger, George Graef, A multi-sensor system for high throughput field phenotyping in soybean and wheat breeding, Computers and Electronics in Agriculture, 128 (2016) 181-192

[12] Zhong Qu and Li Hang, "Research on limage Segmentation Based on the Improved Otsu Algorithm", 2010.

[13] Duarte-Galvan, C., Romero-Troncoso, R., Torres-Pacheco, I., Guevara-Gonzalez, R., Fernandez-Jaramillo, A., Contreras-Medina, L., Millan-Almaraz, J. (2014). FPGA-Based Smart Sensor for Drought Stress Detection in Tomato Plants Using Novel Physiological Variables and Discrete Wavelet Transform. Sensors, 14(10), 18650-18669. https://doi.org/10.3390/s141018650

[14] Vala, M. H. J., \& Baxi, A. (2013). A review on Otsu image segmentation algorithm. International Journal of Advanced Research in Computer Engineering \& Technology, 2(2), 387-389.

[15] Miss Hetal J. Vala, Prof. Astha Baxi, A Review on Otsu Image Segmentation Algorithm, International Journal of Advanced Research in Computer Engineering \& Technology (IJARCET) Volume 2, Issue 2, February 2013

[16] Amruta B. Patil, J.A.shaikh, OTSU Thresholding Method for Flower Image Segmentation, International Journal of Computational Engineering Research (IJCER), Volume, 06, Issue, 05, May - 2016. 